\title{
p21 in chronic and acute liver injury
}

\section{Haksier Ehedego and Christian Trautwein}

p21 historically has been considered a tumor suppressor since first studies showed that $\mathrm{p} 21^{-/-}$mice display spontaneous tumor formation after 16 months and additionally these mice are more sensitive to chemically induced carcinogenesis $[1,2]$.

On the contrary, recently a potential function as an oncogene has been described for $\mathrm{p} 21$. For instance mice deficient for p53 spontaneously develop multiple tumors and an additional deletion of p21 lead to a significant reduction of thymic lymphomas [3].

This argues that the complete spectrum of p21 function during tumorigenesis is not clearly identified. The role of $\mathrm{p} 21$ has been further studied in the $\mathrm{NEMO}^{\Delta \text { hepa }}$ mice model. The NF- $\kappa \mathrm{B}$ pathway regulator NEMO (also known as IKK $\gamma$ ) has been shown to control chronic inflammation and hepatocarcinogenesis in mice.

The hepatocyte specific deletion of NEMO $\left(\mathrm{NEMO}^{\Delta \text { hepa }}\right)$, is of clinical interest as these animals develop a cascade of events which resemble the spectrum of human chronic liver disease, which leads from chronic hepatitis to liver cirrhosis and growth of hepatocellular carcinomas (HCC). Additionally, a recent study using human HCC tissue found a downregulation of NEMO in tumor tissue, further supporting the translational relevance of the $\mathrm{NEMO}^{\Delta \text { hepa }}$ mice model [4].

The deletion of NEMO in hepatocytes triggers increased p21 expression [5,6]. In order to study the relevance of $\mathrm{p} 21$ overexpression for disease progression of $\mathrm{NEMO}^{\Delta \text { hepa }}$ livers, double knockout $\left(\mathrm{NEMO}^{\Delta \text { hepa }} / \mathrm{p} 21^{-/-}\right)$ mice carrying a hepatocyte specific deletion for NEMO and an additional constitutional deletion for p21 were generated.

Although p21 is a cell cycle inhibitor its deletion had no impact on cell proliferation in 8 week-old $\mathrm{NEMO}^{\Delta \text { hepa }} / \mathrm{p} 21^{-/-}$livers compared to $\mathrm{NEMO}^{\Delta \text { hepa }}$ livers. This result was unexpected since p21 binds to $\mathrm{CcnE} / \mathrm{cdk} 2$ and $\mathrm{Ccn} \mathrm{A} / \mathrm{cdk} 2$ complexes thereby preventing progression from $\mathrm{G}_{1}$ - to $\mathrm{S}$-phase. Very likely the loss of $\mathrm{p} 21$ expression is compensated by other cell cycle inhibitors such as p-p27 and $\mathrm{p} 18$.

Despite the unchanged cell cycle activity in p21 deficient $\mathrm{NEMO}^{\Delta \text { hepa }}$ livers, the cell cycle regulator CcnA2 and CcnE2 were overexpressed. However, recent studies discovered that ectopic overexpression of CcnA or CcnE in mouse embryonic fibroblast (MEFs) lead to an increase in DNA double strand breakage [7]. Therefore the enhanced liver injury caused by exacerbation of
DNA damage in p21-deficient $\mathrm{NEMO}^{\Delta \text { hepa }}$ mice could be explained by elevated $\mathrm{Ccn} \mathrm{A} 2$ and $\mathrm{CcnE} 2$ expression. The DNA double strand breakage was quantified by $\mathrm{pH} 2 \mathrm{AX}$ Immunofluorescence staining.

p21 is not only protective against DNA damage in the chronic liver injury model as challenging double mutant $\mathrm{NEMO}^{\mathrm{Ahepa}} / \mathrm{p} 21^{-/-}$mice with Lipopolysaccharide (LPS) enhanced DNA damage massively compared to $\mathrm{NEMO}^{\Delta \text { hepa }}$ mice. After LPS administration $\mathrm{NEMO}^{\Delta \text { hepa }}$ mice suffer from severe liver injury which is reflected in the increased alanine aminotransferase (ALT) and aspartataminotransferase (AST) serum values and apoptotic cells in the liver of these mice. However, in $\mathrm{NEMO}^{\mathrm{Ahepa}} / \mathrm{p} 21^{-/}$ mice transaminases and cell death were significantly enhanced. Finally, this enhanced liver injury in the double knockout animals resulted in a higher lethality of this mice after LPS administration.

The observed hypersensitivity against LPS due to the lack of p21 is mediated via the Tumor Necrosis Factor (TNF), since $\mathrm{NEMO}^{\Delta \text { hepa }} / \mathrm{p} 21^{-/-}$mice which carry in

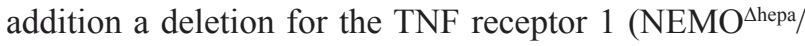
$\mathrm{p} 21^{-/ /} / \mathrm{TNF}-\mathrm{R} 1^{-/}$), showed a strong attenuation of the DNA damage and cell death.

The protective role of $\mathrm{p} 21$ in carcinogenesis was the first time visible in 26 week old knockout animals. Here, the double knockout mice $\left(\mathrm{NEMO}^{\Delta \text { hepa }} / \mathrm{p} 21^{-/}\right)$showed enhanced hepatocyte proliferation as revealed by $\mathrm{Ki} 67$ staining. This resulted consequently into a higher liver weight/body weight ratio but more interestingly p21deficient $\mathrm{NEMO}^{\Delta \text { hepa }}$ livers showed more frequently small tumors in comparison to $\mathrm{NEMO}^{\mathrm{\Delta hepa}}$ livers.

Finally, a significantly increased number of $\mathrm{HCCs}$ were found in 52 week-old $\mathrm{NEMO}^{\Delta \mathrm{hepa}} / \mathrm{p} 21^{-/-}$ animals, meaning that the loss of p21 expression caused exacerbation of hepatocarcinogenesis. Analysing the livers of these mice revealed that only the number of nodules was increased, whereas the sizes of the tumors were not significantly enlarged. This suggests that the loss of $\mathrm{p} 21$ overexpression in $\mathrm{NEMO}^{\mathrm{Ahepa}}$ animals has more impact on tumor initiation than on tumor progression.

Beside hepatocarcinogenesis p21 had an additional protective role in cholestasis. Livers of 52 week old $\mathrm{NEMO}^{\Delta \mathrm{hepa}} / \mathrm{p} 21^{-/-}$animals display yellow inclusions and serum values for alkaline phosphatase, direct and total bilirubin confirmed the cholestatic phenotype. These cholestatic serum markers were significantly lower in $\mathrm{NEMO}^{\Delta \text { hepa }}$ mice. 
Taken together, the enhanced $\mathrm{p} 21$ expression in $\mathrm{NEMO}^{\text {shepa }}$ animals has a protective function in this model, as p21 protects against DNA damage, acceleration of hepatocarcinogenesis and cholestasis. Since liver disease progression is reduced in the presence of $\mathrm{p} 21$ expression, p21 has been shown to act as a tumor suppressor in the $\mathrm{NEMO}^{\text {\hepa }}$ model.

\section{CONFLICT OF INTEREST}

No potential conflicts of interest were disclosed.

Haksier Ehedego and Christian Trautwein: Department of Internal Medicine III, University Hospital RWTH Aachen, Aachen, Germany.

Correspondence: Haksier Ehedego, email hehedego@ ukaachen.de

Correspondence: Christian Trautwein, email ctrautwein@ ukaachen.de

Keywords: DNA damage, hepatocarcinogenesis, liver injury

Received: December 06, 2015

Published: March 04, 2016

\section{REFERENCES}

1. Martín-Caballero J, et al. Cancer Research. 2001; 61(16):6234-8.

2. Topley GI, et al. Proc. Natl. Acad. Sci. USA. 1999; 96(16):9089-94.

3. De la Cueva E, et al. Oncogene. 2006;2 5(29):4128-32.

4. Aigelsreiter A, et al. Hum. Pathol. 2012; 43(7):1012-9.

5. Malato Y, et al. Gastroenterology. 2012; 143(6):1597-1608.

6. Ehedego H, et al. Cancer Research. 2015; 75(6):1144-55.

7. Tane S, et al. Cell Cycle. 2009; 8(23):3900-3. 